\title{
La révision toponymique (et cartographique) en Nouvelle-Calédonie (1983-1993)
}

Jean Chatelier

\section{(2) OpenEdition \\ 1 Journals}

Édition électronique

URL : http://journals.openedition.org/jso/1020

DOI : $10.4000 /$ jso. 1020

ISSN : 1760-7256

\section{Éditeur}

Société des océanistes

\section{Édition imprimée}

Date de publication : 1 décembre 2007

Pagination : 295-310

ISBN : 978-2-85430-010-9

ISSN : 0300-953x

Référence électronique

Jean Chatelier, "La révision toponymique (et cartographique) en Nouvelle-Calédonie (1983-1993) », Journal de la Société des Océanistes [En ligne], 125 | Année 2007-2, mis en ligne le 01 décembre 2010, consulté le 02 mai 2019. URL : http://journals.openedition.org/jso/1020 ; DOI : 10.4000/jso.1020

(c) Tous droits réservés 


\section{La révision toponymique (et cartographique) en Nouvelle-Calédonie (1983-1993)}

par

Jean CHATELIER*

\section{RÉSUMÉ}

Avec l'accord de Nouméa de 1998, impliquant la restauration des noms kanak, la toponymie vernaculaire occupe régulièrement la scène médiatique depuis les premières actions de la province Nord à partir de 2002. Il paraît donc intéressant de voir d'abord, et succinctement, comment ce sujet a été traité depuis les premières cartes officielles jusqu'à nos jours, puis de s'attarder un peu plus sur la révision toponymique menée par le Service topographique de la Nouvelle-Calédonie entre 1983 et 1993, ce en même temps que la révision de la carte IGN de base à l'échelle du 1 : 50 000. Il sera aidé en cela par la Commission nationale de toponymie de l'IGN et l'université Paris III, ex Institut national des langues et civilisations orientales. Cette Action, ou plutôt cette expérience originale et sans précédent, dont on soulignera les principaux traits, a révélé un patrimoine particulièrement riche et abondant, encore aujourd'hui. Si celui-ci est menacé par l'évolution en cours et l'occidentalisation, sa sauvegarde sur le long terme et sa valorisation au travers de la documentation moderne, tant au niveau local que national, voire international, dépendront pour une grande part, de la continuité des actions avec la révision de 1983-1993, de la recherche d'une certaine simplification des écritures au travers d'une normalisation et enfin du respect de procédures d'officialisation. Les compétences en la matière, locales et nationales, n'ont jamais été aussi nombreuses, il conviendrait donc de les rassembler sur cet objectif. C'est le défi qu'il nous faudra, tous ensemble, relever aujourd'hui.

MotS-CLÉS : toponymie, Kanak, langues vernaculaires, archives, cartographie, enquêtes.

\section{SUMMARY}

Since the Noumea agreements in 1998, the revival of Kanak names and of the vernacular toponymy has often been in the light of the media, especially since 2002, when the northern province took the first steps to promote that policy. It is thus of some interest to analyse how this question has been treated, from the first official maps until now, and then to analyse the revision brought to the New Caledonian toponymy by the Topographic Services between 1983 and 1993, at the same time as the IGN revised its maps at scale $1: 50.000$. To do so, the New Caledonian Topographic Services were helped by the IGN National Committee for toponymy, by the University Paris III formerly the National Institute of Oriental Languages and Civilisations. This unprecedented and original experiment has revealed a particularly abundant and rich heritage, which has survived till now, but which is threatened by ongoing evolution and westernization. Thus, its long-term preservation and promotion at local, national and even international levels, through modern techniques of documentation will mostly depend on how the policy carried out between 1983-1993 is continued, as well as on the quest for a simplified and unified writing system and on the respect of official procedures. Local and national skills in such matters have never been so great, they should now focus on that aim. This is the present challenge that must be confronted collectively.

KeYwords: toponymy, Kanak, vernacular languages, archives, cartography, fieldwork.

* Ingénieur géomètre retraité du Service topographique de la Nouvelle-Calédonie (DITTT, déc. 2004), chatelier@offratel.nc 


\section{La toponymie et les langues de Nouvelle- Calédonie}

La dénomination des noms de lieux est une activité aussi vieille que l'humanité et ses premiers balbutiements. Appelée maintenant toponymie, elle ne serait devenue une science qu'assez récemment, à peine un siècle, science qui a pour objet l'étude de la formation et de l'évolution des noms de lieux. Si, dans les anciennes civilisations elle s'est fixée très tôt au travers des premiers systèmes d'écriture, il n'en est pas de même pour de très nombreuses populations du Globe où la tradition orale prédomine encore fortement. C'est le cas de toute notre région Pacifique et donc de la Nouvelle-Calédonie. Selon les archéologues, ces populations océaniennes sont toutes d'origine austronésienne et sont arrivées il y a environ trois mille ans. $\mathrm{Si}$, au niveau polynésien, on retrouve une certaine unité linguistique, il n'en est pas du tout de même pour les Mélanésiens de Nouvelle-Calédonie qui, au contraire, se sont attachés à cultiver leurs différences. Il en résulte une multiplicité de langues. Aujourd'hui, le Lacito-CNRS ${ }^{1}$ ne recenserait pas moins de huit aires coutumières, vingt-huit langues, onze dialectes et certains ont déjà disparu. Si aujourd'hui plus des deux tiers des langues disposent d'un système d'écriture connus des spécialistes (Jacques Vernaudon, comm. pers., septembre 2006), seules quelques-unes d'entre elles disposent d'une certaine tradition d'écriture et d'une production littéraire. D'après Bernard Cerquiglini (2003), « le degré de diversification actuel des langues kanak est comparable à celui des langues indo-européennes ».

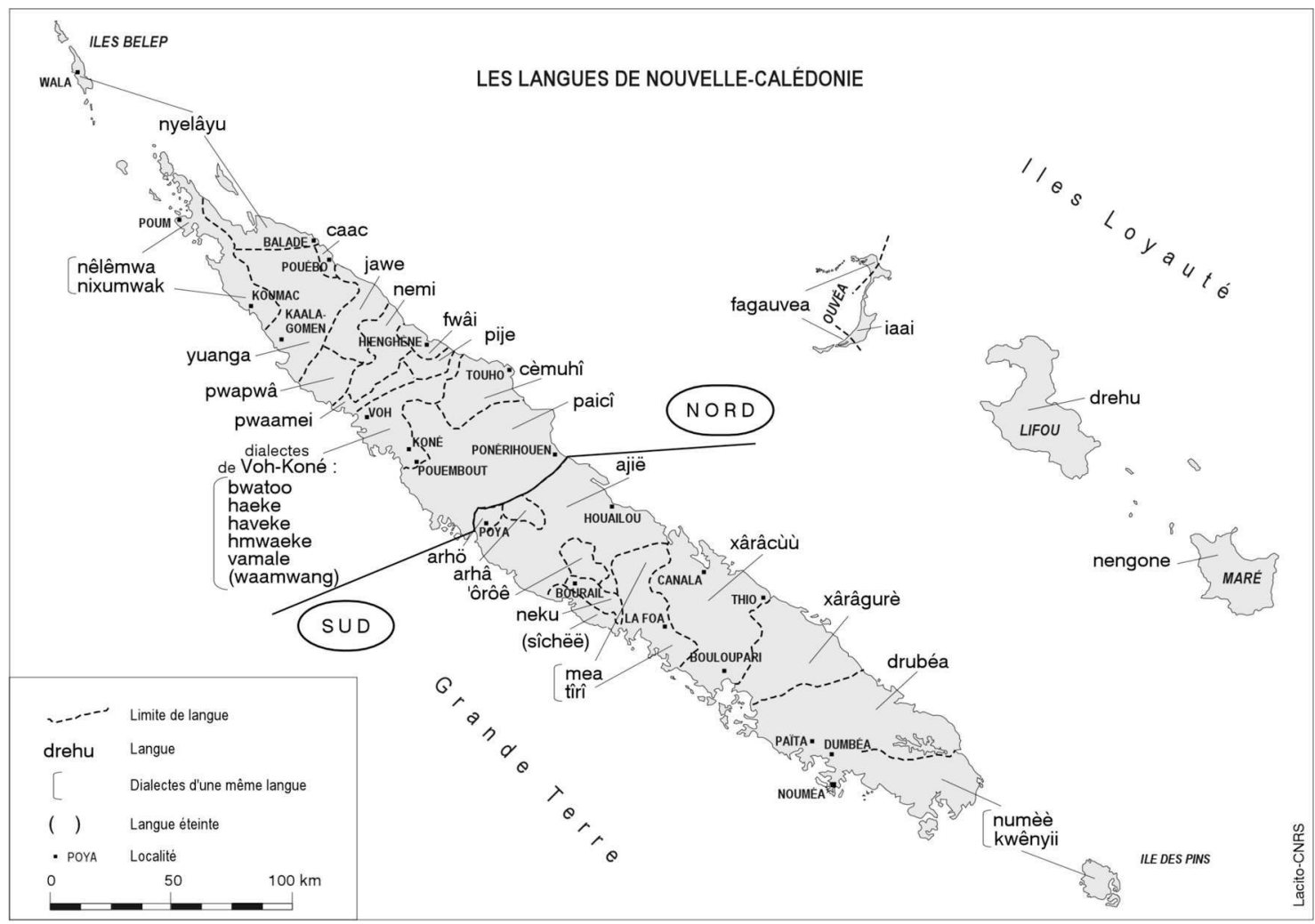

FIgURE 1. - Planche LaCito-CNRS des langues de Nouvelle-Calédonie (avril 2004)

\section{Les premières cartes jusqu'à nos jours}

Cette complexité a fait que la toponymie vernaculaire relevée sur les premières cartes des Européens fut, comme nous allons le voir, relativement pauvre bien que plus abondante que la toponymie européenne, et ce pratiquement jusqu'à nos jours. En excluant celle des premiers grands navigateurs européens, la première toponymie significative fut relevée dès les années 1850 par les hydrographes pour la partie maritime, puis pour la partie terrestre par le Service topographique à partir de 1856 (appelé Service du cadastre jusqu'en 1867), puis, entre 1879 et 1886 , par des officiers de l'Infanterie de marine qui 
entreprirent, au travers de la Mission topographique, la première cartographie générale de la Nouvelle-Calédonie, Loyauté exclues, ce suite à la première insurrection kanak de 1878. Six feuilles furent ainsi publiées à l'échelle 1 : 107000 environ, et ce à partir des nombreux plans au $1: 40000$ (cartes minutes ?). Curieusement, une certaine ressemblance entre les cinq arrondissements créés à cette période et les cinq aires coutumières définies aujourd'hui, pourrait laisser supposer déjà une certaine connaissance des réalités mélanésiennes. Cette toponymie fut officialisée très tôt puis reprise dans « l'orthographe des noms canaques» en 1900. Elle sera ensuite transmise au travers des cartes Laporte (exposition universelle de Paris de 1900), puis Meunier vers 1935, jusque dans les années 1960 (cartes de la Havraise calédonienne)! Suite à la Seconde Guerre mondiale, et à la nécessité de faire évoluer ce territoire, une cartographie générale régulière de la Nouvelle-Calédonie, à l'échelle du 1:50 000, fut réalisée par l'IGN ${ }^{2}$ entre 1951 et 1956, publiée en 1958, 1960 et 1962. La toponymie antérieure fut en partie reprise puis complétée, suivant « les recommandations de l'Institut français d'outremer (Jean Guiart) et des coutumes administratives » (rapport Tual, IGN, 26 octobre 1956), mais très sommairement comme on le verra ci-après.

\section{La révision de la carte IGN et la toponymie}

La carte IGN au $1: 50000$ fut révisée à partir de septembre 1983 et publiée, sauf une, de 1986 à 1994, "série Orange, 4800 », quarante-et-une feuilles, en vente dans les tabacs-journaux. Cette révision proprement cartographique servit de révélateur, puis de moteur à une révision toponymique sans précédent. Ce travail sera mené localement par le Service topographique ${ }^{3}$, de sa seule initiative et sans relation directe avec l'intérêt qui sera porté au monde mélanésien par les instances officielles. Au vu de ce décalage, il n'est pas inintéressant de rappeler quelques événements clés qui accréditeraient l'idée de « l'ignorance constante des réalités locales par les acteurs européens » (Guiart, $2002:$ 75).

«La reconnaissance de l'identité culturelle mélanésienne ", définie dans l'ordonnance $\mathrm{n}^{\circ}$ 82-879 du 15 octobre 1982, donnera lieu à la création de l'Office culturel, scientifique et technique canaque (souvent noté OCSTK). Elle sera précédée par quelques événements significatifs.
C'est ainsi que dès 1974 (fin de période du premier " boom» minier) lors de l'examen de la délibération relative à l'établissement du cadastre en Nouvelle-Calédonie, en fait un inventaire cadastral, quelques élus exprimèrent à l'Assemblée territoriale l'idée d'un "cadastre mélanésien dans les réserves "; des travaux scientifiques conduits par l'ORSTOM furent prévus à cet effet et se dérouleront plus tard en préparation à la Réforme foncière. En septembre 1975 à Nouméa, une manifestation culturelle marquera les esprits par l'ouverture du monde mélanésien à la modernité, ce sera Mélanésia 2000. En 1978, le plan Dijoud envisagera « le développement économique et social à long terme de la NouvelleCalédonie ». Suivront la préparation puis la mise en place de la Réforme foncière en 1980, enfin la création de l'Office foncier en 1982 puis de l'ADRAF, Agence de développement rural et d'aménagement foncier, de compétence territoriale en 1986 et étatique à partir de 1989.

De 1982 à 1984, le Service topographique (sт) entreprit donc, de son propre chef, une recherche systématique de la toponymie d'archive au travers des nombreux plans minutes du ST (près de huit cents) et des cartes marines. Essentiellement d'origine mélanésienne, elle fut très abondante. En janvier 1983 était créée officiellement une commission consultative de toponymie, puis démarrèrent les enquêtes sur le terrain, censées valider la toponymie d'archive et la compléter, l'Office culturel se chargeant de l'enquête, de la partie linguistique et de la transcription en milieu mélanésien. Une première commission communale de toponymie eut lieu en avril de cette même année à La Foa. Les enquêtes se poursuivirent mais fin 1984, il fallut admettre :

1. que cette toponymie d'archive, malgré son importance, trouvait assez peu de résonance auprès des populations, même mélanésiennes ;

2. que les investigations plus directes révélèrent une richesse particulière de la toponymie vernaculaire et, en même temps, une certaine pauvreté de la carte IGN, comme des cartographies générales existantes d'alors ;

3. qu'il fallait surtout prendre enfin en compte l'aspect spécifique de ces langues (par rapport au français) et admettre que l'action de l'oc n'était pas adaptée à la situation et aux délais demandés pour la révision des cartes IGN.

2. Institut géographique national, Paris.

3. Le Service sera provincialisé en 1990. La partie restant au territoire de la Nouvelle-Calédonie, le sT à la DITTT, continuera à traiter la cartographie de base à l'échelle du $1: 10000$ et maintenant $1: 50000$, en participant aussi aux cartes marines (du domaine de l'État). 


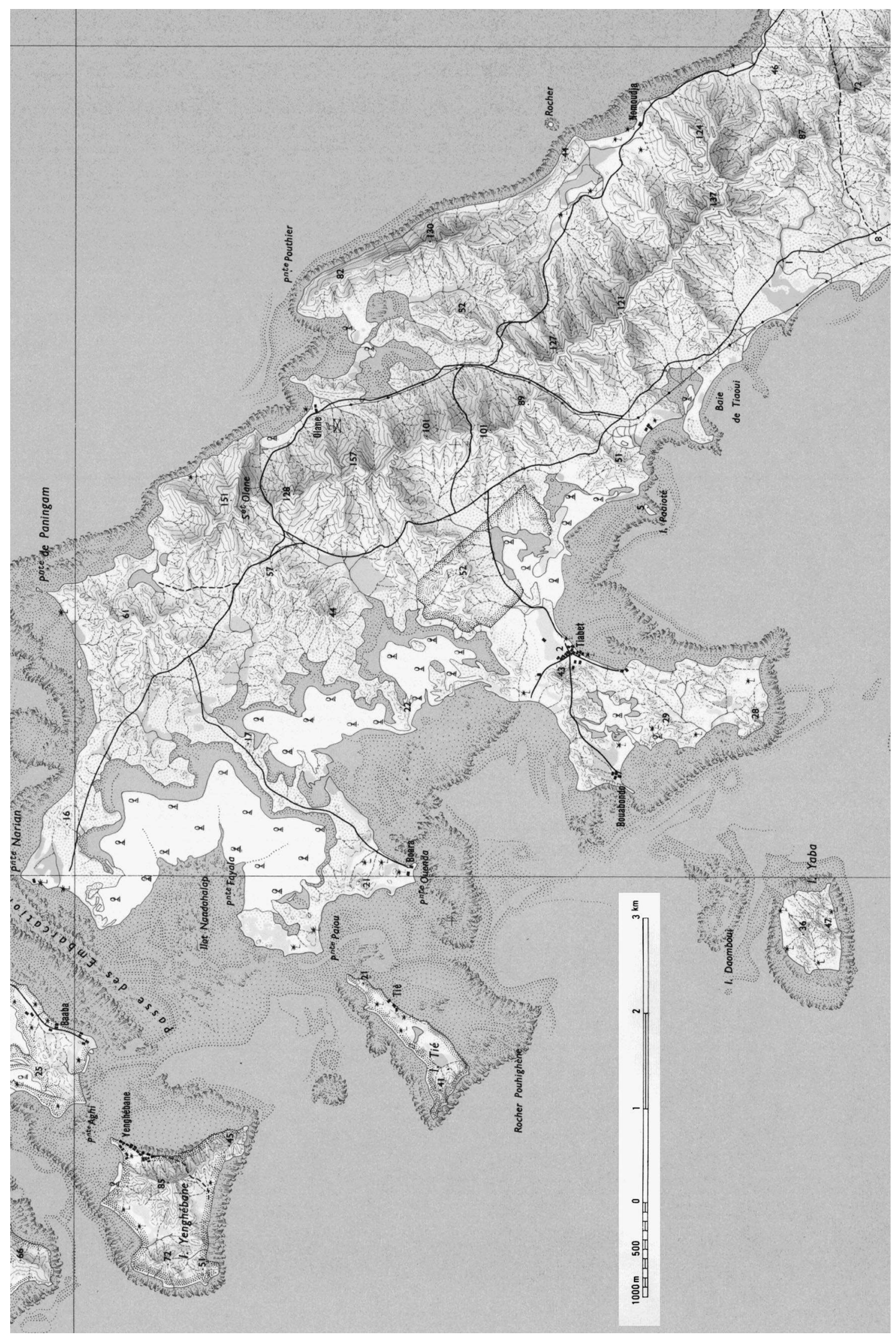

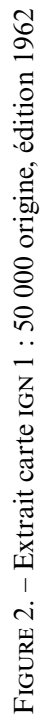




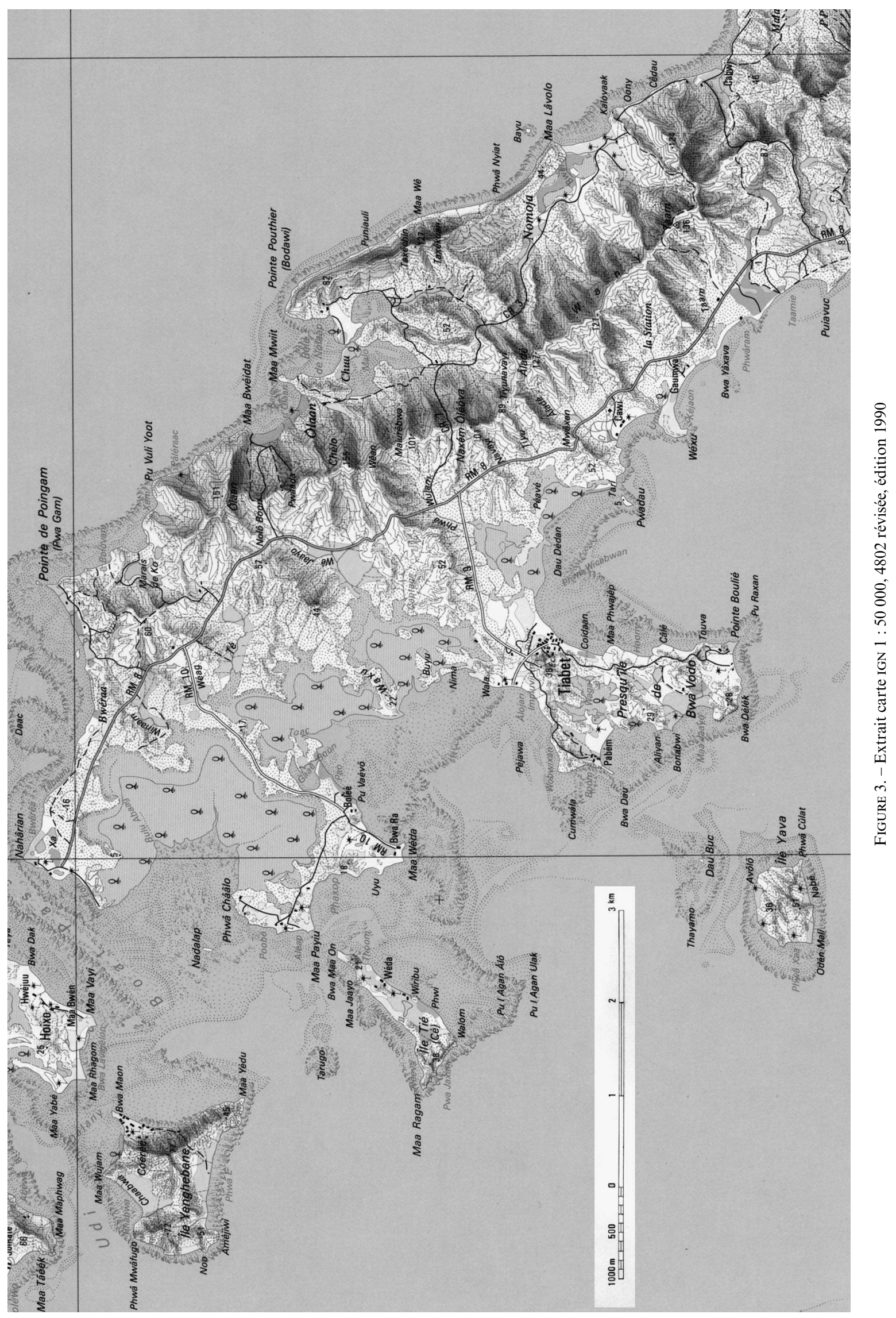


Deux commissions officielles eurent encore lieu en 1984, l'une à Lifou en juin, puis l'autre à l'île des Pins en novembre. Mais les Événements de novembre 1984 provoquèrent l'interruption de ces commissions, qui dure encore et n'a jamais fait l'objet de la moindre explication. Malgré le climat difficile qui suivit pendant les années consécutives aux Événements, les enquêtes continuèrent sur le terrain, en parallèle et en sus de la révision cartographique proprement dite, le seul objectif qui sera officiellement déclaré.

\section{L'importance de la toponymie vernaculaire et sa prise en compte}

En 1985, des évolutions décisives se firent. D'abord, une expérience tout à fait fortuite et révélatrice eut lieu à Moindou, une région peu peuplée, a priori vide de toponymes; ce que l'on pensait aussi d'une bonne partie de la Grande Terre, à la différence des îles. C'est à la tribu de Kèrè que, sous une pluie battante, une équipe de révision trouva refuge dans une cabane et qu'elle rencontra un Mélanésien qui ramenait ses filets. En moins d'une heure, il nommait et situait sur la carte IGN, près de quatre-vingts toponymes entre Moindou et Bourail, dont une bonne partie en français qui s'avèrera peu connue des Calédoniens de la région mais dont lui avait gardé le souvenir. Il s'agissait de Jacques Sanon, petitchef de la tribu de Moméa, alors commandant au RIMAP (Armée de terre). Quelques mois après, on poursuivit ce recueil avec lui dans la région entre Moindou-Bourail et la tribu de Table Unio. Ce sont près de trois cent toponymes qui furent ainsi recueillis en une dizaine de jours. Il fallait alors admettre que la toponymie vernaculaire touchait, en abondance, tout aussi bien la Grande Terre que les îles Loyauté et autres îles.

Il fallait aussi faire comprendre aux géomètres et responsables que les langues mélanésiennes étaient des langues à part entière, même les langues restées orales, les plus nombreuses, avec une grammaire, un dictionnaire; comprendre aussi que ces langues avaient leurs spécificités, en particulier phonétiques, et que l'on ne pouvait pas continuer à se contenter de francisations approximatives. C'est pourtant ce qui s'était passé jusque-là, depuis l'origine de l'implantation européenne. C'est ainsi que le professeur et ethnologue à Nouméa, Jean Guiart, rédigeait en 1956, une notice à l'attention des géomètres du ST et de l'IGN pour la «transcription des noms toponymiques indigènes [afin de] mettre un peu d'ordre dans ce chaos » des écritures, sans règles, tout en y conservant un peu le sens. Elle restera un vœu pieux pour le Service topographique, et d'un effet limité pour la mission IGN, car il eût été quasiment impossible aux opérateurs IGN de mener les enquêtes qui auraient été nécessaires dans le temps qui avait été fixé pour la mission. Citons malgré tout un extrait de l'introduction de Jean Guiart :

" [l'écriture] des centres européens obéit à la fantaisie la plus grande [...]. Il y a Plum (prononcez Ploum), Doumbéa devenu Dumbéa, Yengène puis Hienghène, Koumac mais aussi Couli. Pourquoi?»

Le recueil de la toponymie d'archive révélera l'ampleur de la situation et donc l'inconsistance de la "coutume administrative» dont parlait l'IGN en 1956. C'est ainsi que, suivant les plans et sur plus d'un siècle, un même élément géographique pourra comporter jusqu'à cinq à six écritures différentes.

Mais il fallait aussi reconnaître la complexité du problème pour des géomètres sans formation spécifique. Et Jean Guiart d'ajouter :

« les sonorités des dialectes de la Grande Terre sont souvent déconcertantes à nos oreilles [...]. Il en résulte que pour entendre correctement un nom indigène, il faut non seulement faire un effort de volonté, mais aussi savoir à l'avance à quels sons il est possible de s'attendre. »

L'approche avec des linguistes, à partir de 1983-1984, marquera donc un progrès décisif sur cet aspect. Progrès compromis ces dernières années par des approches particulières sans continuité et par un certain désintérêt pour la question des responsables publics, confortant ainsi, sans doute, «leur ignorance constante $[\ldots] »$.

En août 1985, on fit suivre aux géomètres un stage d'une semaine pour les sensibiliser aux langues mélanésiennes, stage conduit par des linguistes kanak du CTRDP ${ }^{4}$. Si les premiers jours furent marqués par quelques sourires plus ou moins ironiques - leur a priori n'était pas favorable et ils découvraient un vocabulaire quelque peu ésotérique (à leurs yeux) : soufflerie, occlusives cacuminales, rétroflexes sourdes ou sonores -, il n'en fut plus du tout de même dans les derniers jours. Ce fut pour tous les participants un nouvel eurêkà, une véritable révélation. Sortant de la torpeur induite par les enquêtes réalisées à partir des seules archives, ils voulurent en découdre et repartir au plus vite sur le terrain. Ce fut un franc succès. Les linguistes ne pouvant pas se déplacer sur le terrain, et comme il n'était pas question de les remplacer, c'est en janvier 1986, et toujours à Moindou, que fut réalisée la première enquête enregistrée au magnétophone.

4. Centre territorial de la recherche documentaire et pédagogique. 


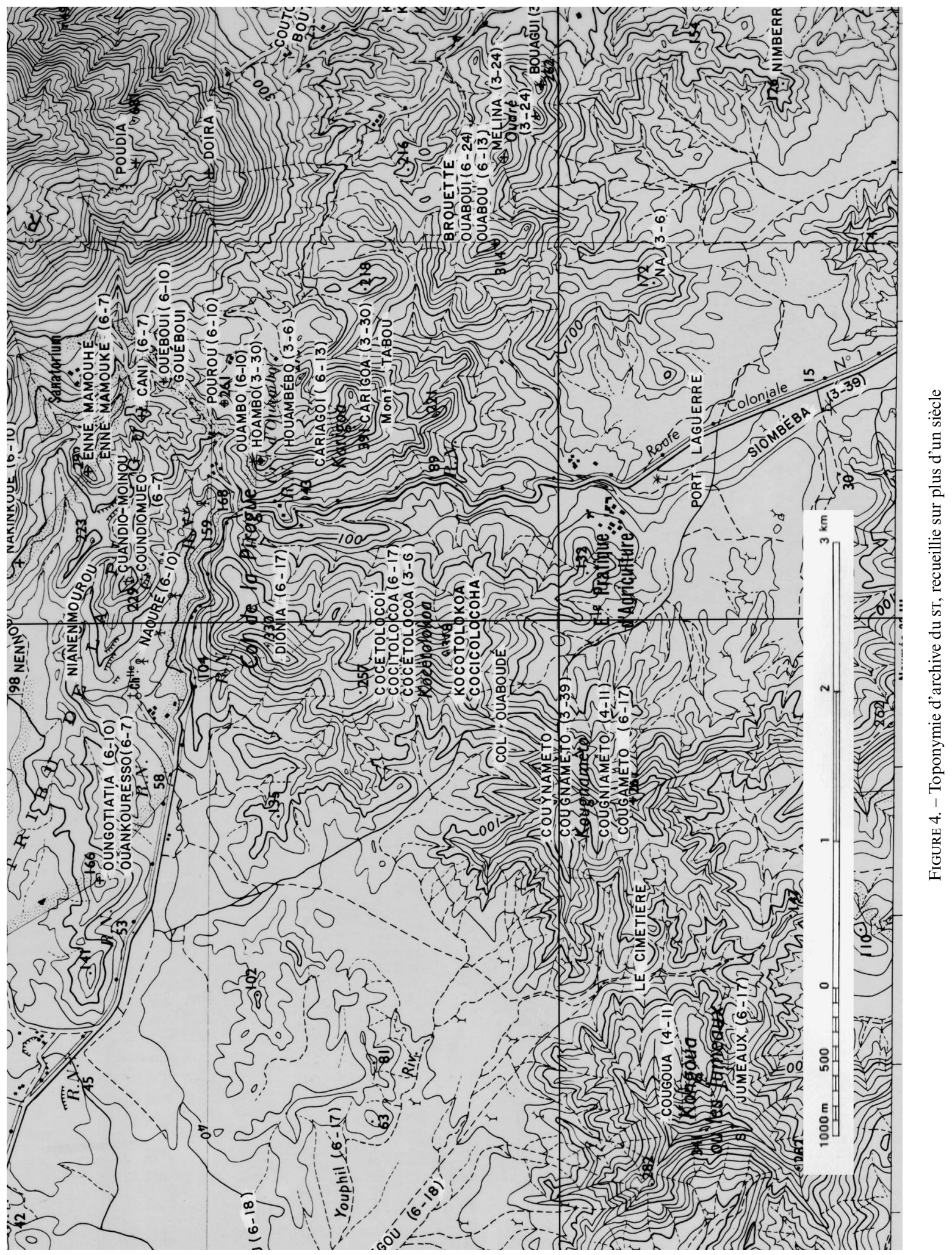


Mais il fallut encore plus d'un an pour que les géomètres disposent d'appareils de service. L'enregistrement était important pour ne pas retarder les investigations et surtout la révision des cartes, l'objectif premier et officiel. Les linguistes se déplaceront à la fin et ceci améliorera beaucoup la qualité des enquêtes et de la saisie phonétique. Autre point important, la nécessité de respecter la manière de faire et l'organisation du monde mélanésien, en précédant l'enquête d'une petite coutumes et en laissant aux clans le soin de nommer leurs représentants pour le recueil de l'information dans leurs zones d'influence coutumière. Ce petit geste d'introduction sera sans doute une des clés essentielles de la confiance qui nous sera accordée pratiquement partout, et de l'abondance de l'information recueillie, et ce malgré le climat tendu de l'époque. Ces méthodes seront par ailleurs appliquées avec succès par le SHOM $^{6}$ aux îles Marquises en 1987-1988. Au niveau de la transcription, l'alphabet phonétique international fut utilisé assez longtemps, mais il ne pouvait naturellement suffire, aussi les linguistes se rabattaient-ils généralement sur les quelques langues écrites les plus proches en affinité. Malgré tout, on décida qu'il fallait convenir d'une certaine homogénéité des écritures cartographiques, au moins sur la Grande Terre, comportant encore assez peu de langues dotées d'un système d'écriture. C'est ainsi, qu'entre 1988-1990, monsieur Une Unë, détaché du CTRDP au ST, établit une convention phonétique pour la transcription des langues mélanésiennes de Nouvelle-Calédonie. Il le fera en collaboration avec la Commission nationale de toponymie à l'Ign (Paris) et l'INALCO'. Bien que tardive, seulement appliquée à partir de 1988, la toponymie antérieure sera entièrement retranscrite et utilisée dans toute la cartographie de base au 1:10 000, puis 1:50 000 du Service topographique de la Nouvelle-Calédonie, ainsi que sur les cartes marines. La convention distingue les deux grands groupes classiques, les Loyauté et la Grande Terre, et marque bien la différence avec le français avec la distinction des sons, les uns communs, les autres, spécifiques aux langues mélanésiennes. Après un arrêt de trois à quatre ans, deux dernières enquêtes furent menées en 1993 par un opérateur spécialement formé, puis détaché pour la transcription directe des toponymes suivant la convention INALCO.

Ces enquêtes publiques, menées de 1983 à 1993, couvriront pratiquement l'ensemble de la
Nouvelle-Calédonie. C'est ainsi que la base informatique du ST/NC comporte aujourd'hui plus de 18000 toponymes. De la sorte, la toponymie de la carte IGN au 1:50 000 sera multipliée par trois, passant de 4100 à près de 12000 , à laquelle s'ajoute celle, hors coupures, portée sur les cartes marines et cartes IGN au 1:200 000 . Sachant au surplus que la même carte, en France, comporte une densité au moins deux à trois fois plus élevée, c'est la confirmation du vide existant antérieurement même pour une densité de population bien plus faible. Mais le travail n'est pas complètement terminé !

\section{L'expérience vécue et la force de l'oralité}

Ces enquêtes ont aussi révélé un monde en grande partie inconnu des Calédoniens et même maintenant d'une partie de la jeunesse mélanésienne. Un monde inconnu mais aussi souvent passionnant, parce qu'on a dû réaliser, parfois à nos dépens, que la toponymie, au travers de nos populations à traditions orales, recouvrait en fait une réalité particulièrement forte, une réalité que nous Occidentaux avons perdue avec l'écrit. C'est en juin 1984, lors de la deuxième convention officielle de toponymie à Lifou, au travers des envolées lyriques des coutumiers et des pasteurs, que notre premier déclic se produira :

« la toponymie, c'est notre histoire, notre géographie, notre cadastre, nos récits, nos mythes et légendes, nos migrations, notre identité personnelle et clanique $[\ldots] »$

Cette réalité deviendra une évidence de plus en plus grande au fur et à mesure de nos enquêtes et nous serons souvent frustrés de ne pas avoir le temps (ni les compétences) pour aller plus loin. Puis on connaîtra quelques moments d'émotion au terme de certaines enquêtes, avec ces vieux qui se sont mis à pleurer, non parce qu'on les avait quelque peu « malmenés », et ce fut parfois le cas (il fallait conclure dans les temps), mais parce qu'ils avaient pu, enfin, léguer leur savoir. Ils étaient soulagés d'avoir transmis leur héritage, mais un héritage en partie oublié, voire parfois méprisé, en particulier par la jeunesse, tournée de plus en plus vers le modernisme et son immense miroir aux alouettes. On découvrira aussi les toponymies de guerre (Paita) pour se situer dans les espaces rivaux convoités, les injurieuses (Ouinané/Tontouta), toujours à l'encontre de clans voisins pas toujours appréciés, par-

5. NDLR. Présents (étoffe, tabac, monnaie...) faits et paroles prononcées lorsqu'on arrive quelque part en signe de respect envers les maîtres du lieu et leurs ancêtres.

6. Service hydrographique outremer.

7. Institut national des langues et civilisations orientales, Paris, connu aussi sous le nom de « Langues'O ». 
fois des toponymies d'archive grossières qui provoqueront l'hilarité de nos informateurs (Bourail, Arama) ; sans doute les traces de quelques rancœurs à l'encontre d'un géomètre un peu trop exigeant. Sur Katrikoin, ce sera un véritable enchevêtrement de toponymies qui traduit probablement une zone de passage importante ou d'occupations successives. Cette toponymie marque aussi, parfois douloureusement, les anciennes luttes et rivalités entre les clans puis les déplacements et conflits provoqués par la présence et l'implantation européenne. Les récits fantastiques où l'imaginaire et le réel sont étroitement liés, sont aussi une expérience un peu troublante au départ pour un interlocuteur non averti, mais aussi une marque de confiance que nous nous sommes attachés à respecter. Autre point qui nous a frappé, c'est l'aisance avec laquelle évoluaient très souvent les informateurs au travers de nos cartes, puis leur impressionnante mémoire des récits, des événements, mémoire des lieux, des mythes et légendes : tout ce qui constitue le socle de leur tradition orale. C'est une expérience encore plus forte que fera le linguiste Alexandre François du Lacito-CNRS, auprès d'anciens de Vanikoro, 217 ans après le naufrage du Français, dans la recherche de Lapérouse en mai 20058. Quant au Père Dubois ${ }^{9}$, il parlera de « coup de foudre » dans ses premières expériences d'ethnologie en 1935 dans le Nord Sinaï, où accompagné d'un Dominicain, ils demandèrent «à un bédouin, sorti du néant et du sable, où se trouvait Eilat (Elath). Il nous montra un désert sablonneux, le lieu-dit exact conservé (dans la tradition orale) depuis le roi Salomon (3000 ans !) ».

Les enquéteurs ont aussi été intrigués par le fait que la toponymie européenne était bien souvent mieux connue par les Mélanésiens que les Européens; et les Mélanésiens n'ont jamais hésité à nous la donner. Qui connaît par exemple la rivière des Malabars en bas du Col de la Pirogue, tribu de Saint-Laurent, le Pont des amoureux un peu plus loin, à la sortie de la Tamoa, le Stop et le Tableau toutlemonde (créole) dans l'ancien col de Boghen ? Ceci démontre, une nouvelle fois, l'importance persistante, de l'oralité en milieu mélanésien.

\section{Un patrimoine en perdition}

Malgré tout, il faut bien admettre que ce patrimoine, s'il n'a pas été recueilli à temps, s'en va à

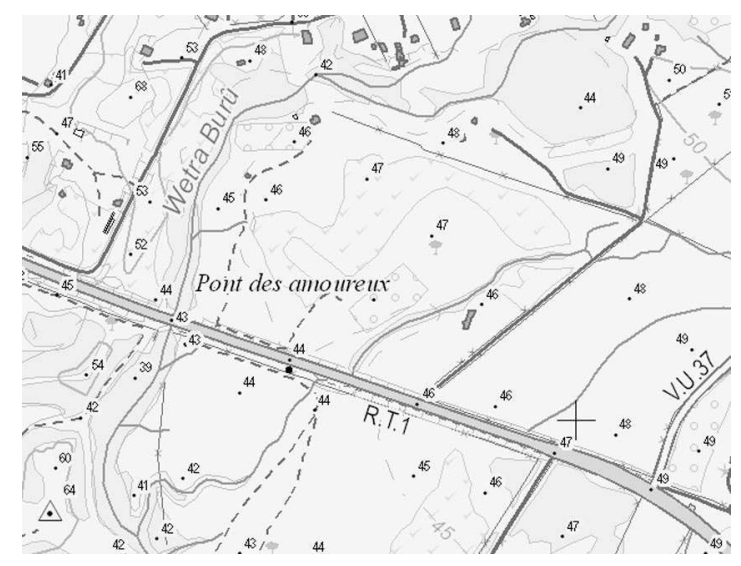

Figure 5. - Le Pont des amoureux, à la sortie de la Tamoa (C. Païta, 1987)

jamais avec la disparition irrémédiable des porteurs de tradition et aussi l'européanisation. Si la Mission topographique note la présence de très nombreux sentiers canaques, et donc d'échanges entre les populations, principalement Est Ouest, la présence européenne et les mouvements de populations les réduiront de manière décisive. L'importante toponymie d'archive, restée sans écho au cours de nos enquêtes, est là pour le prouver au-delà du simple aspect de la diversité « des écritures sans règles ». Les enquêtes du Sud l'ont démontré : très peu de toponymie a pu être recueillie vers l'intérieur des terres, alors que l'on sait qu'il y en avait. Ainsi en est-il de la toponymie détaillée, recueillie par le lieutenant Bourgey en 1864 , lors de son voyage d'exploration de Ounia à Boulari. Il tentera aussi de relater au travers du parcours et les récits de ses aides, « les drames historiques [...] migrations [...] du peuple calédonien [...] qui n'écrit son histoire ni par des monuments, ni par des manuscrits». Autre cause de disparition, la mobilité des populations mélanésiennes et leur accès à la culture européenne, entraînant la désaffection progressive des langues mélanésiennes au profit du français. Ainsi, sur la base du recensement de 1996, sur les $44 \%$ de Calédoniens que représentait la population mélanésienne, $38 \%$ ne parlaient déjà plus leur langue.

Quelques mots sur la toponymie procurée par les chercheurs et scientifiques, parfois importante. Ce fut une des premières pistes explorées mais vite abandonnée car souvent inexploitable sur le plan cartographique : difficulté de connaissance et d'accès aux diverses documentations, transcriptions souvent spécifiques, difficulté à trouver les sources (informateurs, date...), 
et surtout non prise en compte des aspects particuliers de la saisie cartographique et de sa destination publique (dans l'intérêt général et actuel) - voir ci-après " La carte officielle... ». C'est donc, en partie, encore de l'information perdue et on ne parlera pas ici des travaux préparatoires à la Réforme foncière, classés confidentiels au ministère de l'Outre-Mer. Au vu des multiples publications et approches en cours, il serait souhaitable que scientifiques et cartographes se rapprochent et participent ainsi à l'enrichissement et à la pérennisation d'un patrimoine exceptionnel en la matière.

\section{La véracité de l'information recueillie. Les conflits en sommeil}

Aucun enquêteur ne parlant la langue, et elles sont nombreuses, une question s'est régulièrement posée à nous : pouvons-nous avoir été leurrés ? C'est tout à fait possible mais nous n'avons jamais eu ce sentiment et il y a des indices de sincérité qui ne trompent pas. Aux îles Loyauté, les enquêtes qui ont été menées avec des gens maîtrisant la langue n'ont fait apparaître aucun désaccord. Puis nous avons retrouvé, comme à Maré où la notation était assez fidèle, tous les toponymes notés par le révérend père Dubois, plus de trente ans auparavant. Des reprises récentes, à d'autres endroits, ont confirmé la véracité des informations recueillies en 1985 et 1987. Par contre, et malgré les dix ans d'enquête, tous les clans n'ont pu être consultés partout et ce que chacun considère comme sa «zone d'influence coutumière » et ses désignations peuvent varier d'un clan à l'autre et donc aussi l'information recueillie. C'est ce que nous avons pu constater, par exemple chez les Belep, entre l'enquête faite auprès des réfugiés du MontDore et celle faite sur place plus tard. Là, aucun consensus n'a pu encore être établi. Il y a aussi le fait que les enquêteurs se présentaient avec un objectif relativement neutre: "prendre en compte la toponymie sur les nouvelles cartes $\gg$. Il n'en serait pas de même aujourd'hui avec les très gros enjeux miniers, voire touristiques, en cours, où on peut craindre le réveil d'appétits soudains, et donc de désignations particulières par des gens qui ne sont pas nécessairement les « ayants droit coutumiers ", comme cela a pu être constaté au Vanuatu après l'indépendance et comme on peut le voir aujourd'hui en NouvelleCalédonie. Tenant compte des limites de ces enquêtes et de la contestation toujours possible, une certaine traçabilité a été réalisée par diverses mentions, comme celles des enquêteurs et infor- mateurs (clans) - voir ci-après -, en attendant qu'un certain «droit coutumier foncier » sur la qualité juridique des clans terriens et des personnes soit établi et porté à la connaissance de tous les responsables en la matière.

Autre indice de véracité, lorsque les premières cartes IGN révisées sont apparues, telles celles de Lifou en 1986, les réactions des aides géomètres originaires de l'île furent assez édifiantes : ce n'était plus la carte du gendarme ou du médecin (du Blanc), c'était leur carte! Ils se reconnaissaient dans tous les noms portés, et avec beaucoup de surprise et d'intérêt. Mais on a pu aussi en sentir les limites, car certains aides étaient de districts différents, aussi sur « les frontières » on a pu vérifier, à nos dépens, que certaines animosités entre clans étaient encore vives par rapport aux noms portés. Il ne fallait pas insister. Sur Maré, ce fut encore plus direct. Lorsqu'on s'adressa au maire pour lui demander si on pouvait commencer par porter les limites de district, comme à Lifou, celui-ci s'est exclamé : "Mais vous voulez la guerre? ». Le sujet était sensible et on ne porta que les noms. En dehors du grand district de Guama, où il existe des registres coutumiers, donc des écrits, il fallait admettre que dans les autres districts l'oralité ne suffisait plus à assurer la conservation historique des accords et échanges fonciers successifs, même si ceux-ci ont vocation à être mouvants et re-négociables à tout moment. Il faut aussi noter la forte interférence des limites de district, dites « administratives ».

\section{Les aspects particuliers de la saisie cartographi- que et restrictions}

La spécificité de l'approche cartographique est aussi un aspect essentiel de la toponymie. L'enquêteur est de préférence un géomètre, il est initié aux méthodes d'enquête et aux particularismes mélanésiens. Il doit préalablement se munir des documents cartographiques nécessaires : photos aériennes (vision en relief), plans, ortho-photos, etc. Au moment de l'enquête, un certain nombre d'éléments sont à prendre en compte. D'abord, il faut saisir toutes les données utiles à la traçabilité de l'enquête. Indépendamment des contestations toujours possibles, comme nous l'avons vu, l'enquête pourrait être complétée, mise à jour, reprise et il est important de disposer au moins des dates et lieux d'investigation, des enquêteurs (et qualité), des informateurs (clans), de la langue parlée, de l'enregistrement au magnétophone, des observations éventuelles et si nécessaire d'un compte rendu. 


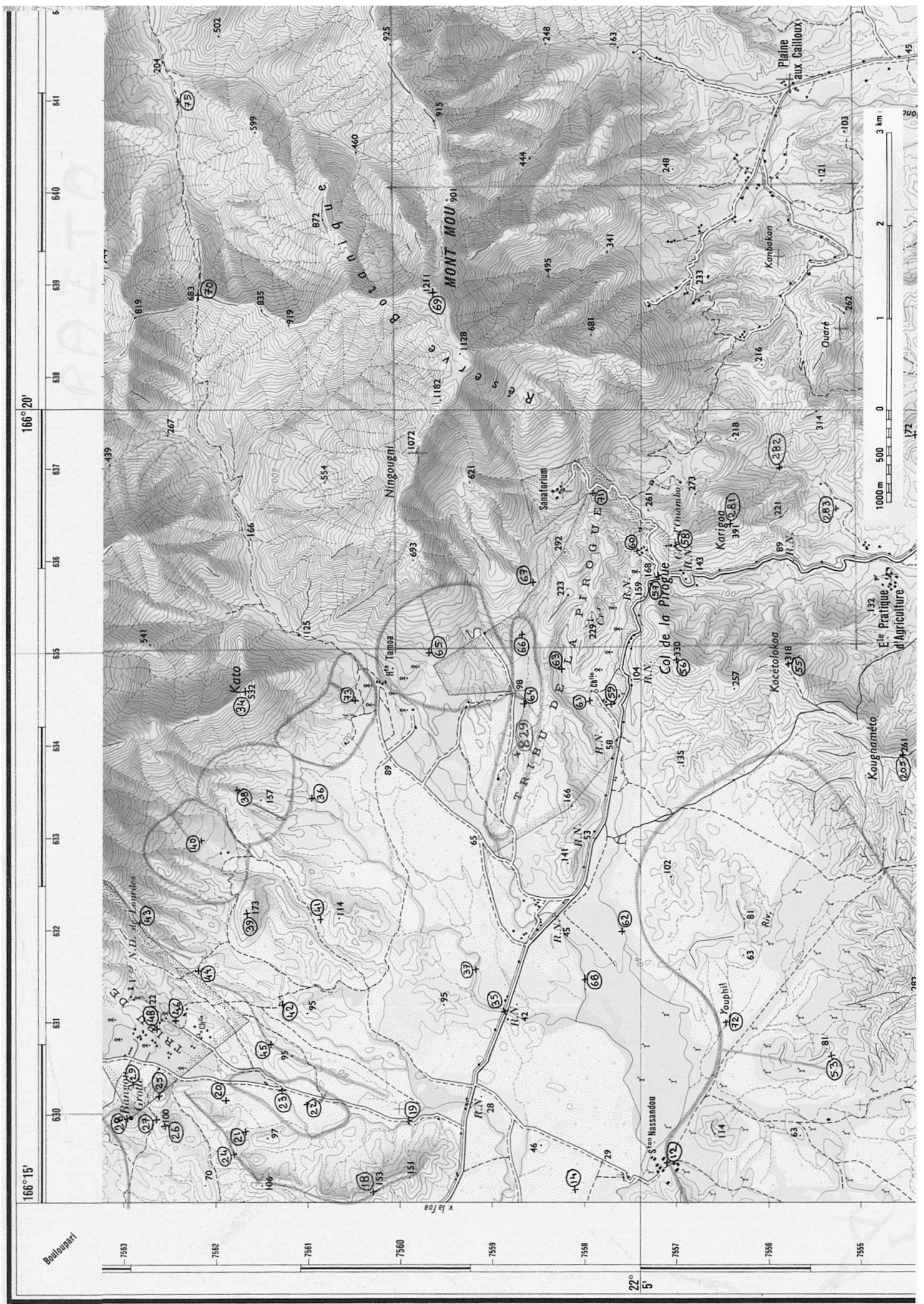

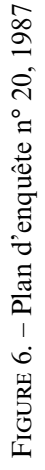




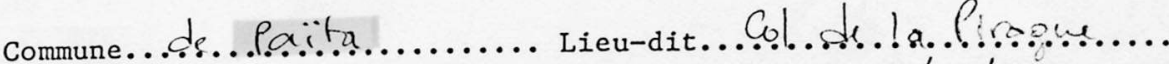
Géomètre :....H.A.T.L!ER..FFNG........ Date...!9...96/.8.7.

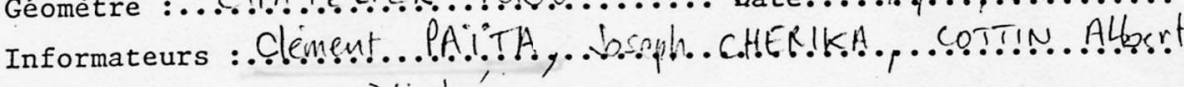
Langue ou dialecte :. Düblos s.... Transcripteur $: \ldots \ldots \ldots \ldots \ldots \ldots \ldots \ldots \ldots$ Orthographe

Enregistrement Locuteurs................ Observations :

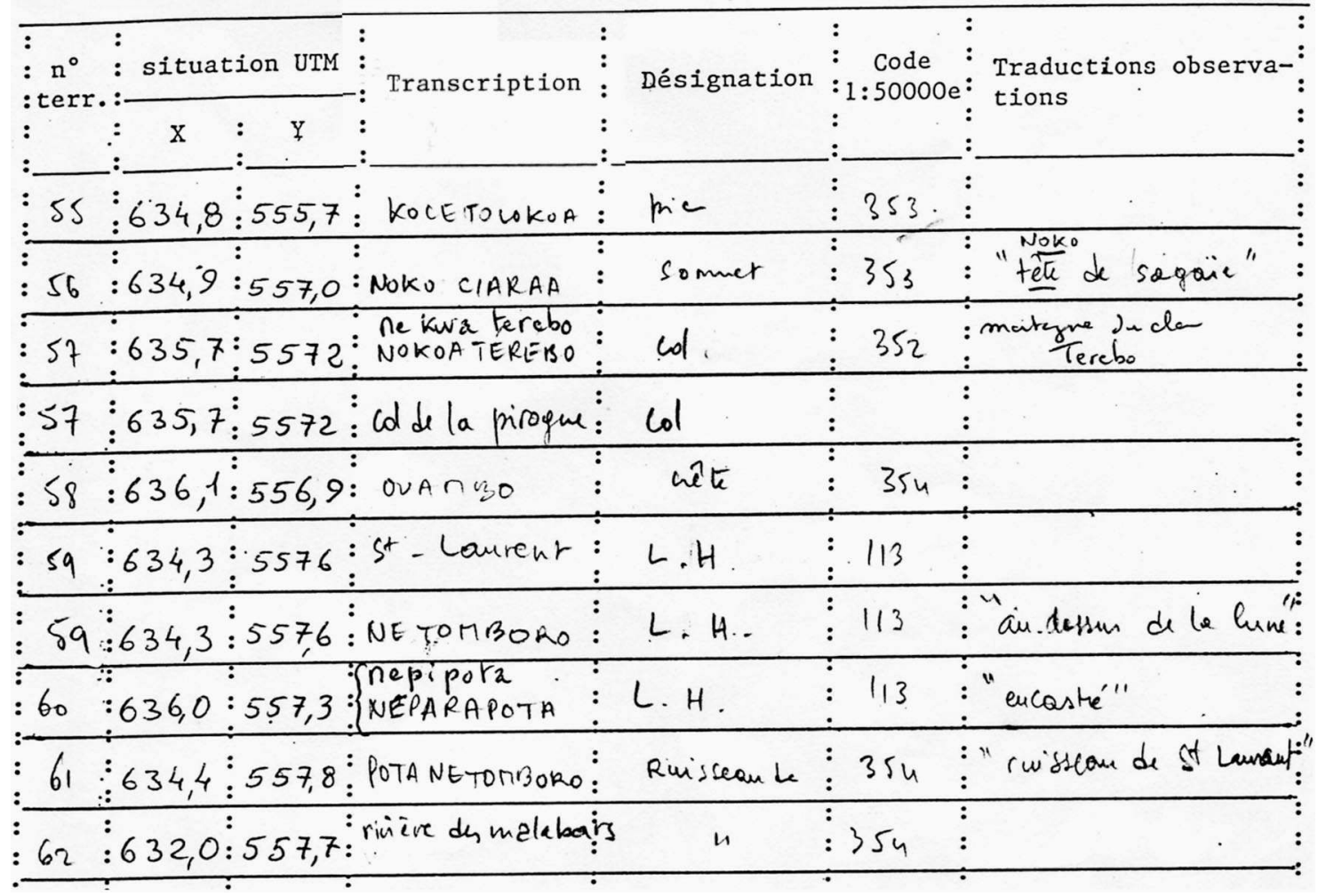

Figure 7. - Extrait cahier d'enquête nº 20,1987

Soulignons au passage que ce problème de traçabilité est une des raisons essentielles du rejet de la toponymie fournie par les chercheurs. Ces préalables étant acquis, il faut qu'on puisse saisir l'importance du toponyme pour le traduire sur la carte par des tailles d'écritures et des dispositions particulières. Il y a aussi sa nature topologique : est-ce un point (sommet, pointe), une ligne (rivière, route, sentier), une surface, une zone plus ou moins grande (plateau, vallée, bassin) ? On y ajoute aussi généralement un point d'ancrage, défini en coordonnées, qui permet au rédacteur principal, et autres rédacteurs ou rédacteurs successifs, par exemple des mises à jour ou réfections ultérieures, de bien placer son toponyme au travers des autres objets mais sans trop s'éloigner de ce point : problème de continuité dans le temps, de lisibilité et de rendu artistique. $\mathrm{Au}$ moment de l'enquête, on visera l'échelle la plus grande qui comportera donc le maximum de détails et de toponymes, mais sans perdre de vue les autres échelles plus petites qui pourront résulter de la généralisation où on éliminera les toponymes les moins importants, par 
exemple du 1: $10000(10 \mathrm{~cm}=1 \mathrm{~km})$ au $1:$ $50000(2 \mathrm{~cm}=1 \mathrm{~km})$. Ce n'est pas toujours un exercice facile, ainsi est-il essentiel que cet aspect ait été pris en compte au moment de l'enquête. Par ailleurs, et quand on connait la propension des Mélanésiens à tout nommer, il a fallu régulièrement rappeler aux informateurs qu'on ne pouvait prendre en compte une toponymie trop domestique, voire privée. Quant à la toponymie historique, elle a été régulièrement demandée, exceptionnellement prise en compte au niveau des cahiers d'enquête mais pas sur la carte qui est elle, répétons-le, destinée aux gens d'aujourd'hui.

Une des qualités essentielles de l'enquêteur qu'il faut aussi souligner, c'est sa capacité d'écoute, car il faut bien comprendre et surtout bien situer géographiquement le toponyme, ce qui n'est pas toujours facile, étant donné que pour des raisons pratiques les investigations se font le plus souvent "en chambre» et donc que les parcours sur le terrain ne sont pas toujours possibles. Une fois le processus engagé avec le ou les informateurs: ne pas poser la question avec la réponse (un grand classique) et surtout sentir le ou les moments où il y a presque un total accord dans le cheminement géographique et la compréhension réciproque. Revenir au moment opportun par le rappel de quelques repères ou indices connus où on peut reprendre le fil commun. Un certain silence s'installe alors. Quant à la magie dont parlent les collecteurs de tradition et Alexandre François, le soir au coin du feu, on n'y a malheureusement pas eu droit, mais on n'a pas à se plaindre. Il faut aussi parler des toponymes mal situés qui perdent une partie de leur valeur, c'est une source de confusion pour l'utilisateur, voire de conflit entre clans, comme nous avons pu le constater à Maré avec quelques toponymes du révérend père Dubois.

Une particularité aussi de la toponymie vernaculaire, c'est l'absence d'équivalence aux termes maritimes occidentaux tels que : baie (Opegejë $\mathrm{Ne}$ We, Baie de Châteaubriand à Lifou), cap, presqu'île, passe, mer, sauf du coté des Bélep. Mais ce sujet n'a été qu'effleuré, comme sa réciproque.

Dire que ces principes ont toujours été respectés serait bien prétentieux, compte tenu de la diversité des intervenants, de l'étalement des enquêtes sur dix ans et des nombreuses difficultés rencontrées.

\section{« La carte officielle », une affaire publique et des obligations légales}

Dans l'esprit du public, y compris des scientifiques et responsables publics, la "carte officielle », c'est la carte IGN à l'échelle du 1:50 000, les cartes marines et accessoirement celles du Service topographique au 1:10 000. Celles-ci constituent, comme nous l'avons vu, les « cartes de base » de la Nouvelle-Calédonie. Mais au niveau toponymique, et mis à part les cartes marines et les cartes IGN, considérées encore parfois comme la «carte d'état-major», ce n'est plus tout à vrai dans la mesure où il existe des procédures d'officialisation qui, pour la Nouvelle-Calédonie, n'ont été mises en œuvre qu'en 1983-1984. Il s'agit des commissions consultatives de toponymie. Elles seraient à réactualiser après la loi organique de 1999, au travers des compétences partagées, officielles ou officieuses.

Comme ces cartes sont faites «pour les gens d'aujourd'hui et pour le plus grand nombre », la toponymie n'est pas qu'une affaire de linguistes, d'ethnologues ou de spécialistes de la culture, c'est un outil utilisé par un grand nombre de gens, dont bien d'autres scientifiques, des professionnels et encore plus des responsables publics, aussi dans la plupart des pays la toponymie faitelle l'objet d'une nomenclature officielle et évolutive. De plus, l'usager est très sensible à l'écriture des noms sur la carte et à leur pérennité, surtout dans sa région; d'après l'IGN, ce point constitue l'essentiel des critiques formulées à l'égard de la carte. Ici le problème est en partie occulté par le fait que la cartographie "nouvelle » semble encore très peu connue du monde mélanésien qui lui préfère sans doute l'oralité. En France, l'expérience est grande et ancienne au travers de la Commission nationale de toponymie (IGN-Paris) et ces dernières années, les choses ont encore bien évolué avec la prise en compte des langues régionales et de normes face à la mondialisation des échanges en matière d'information géographique. Par ailleurs, si la DGLFLF ${ }^{10}$ classe les langues mélanésiennes parmi les langues de France, il resterait cependant à réaliser leur prise en compte au niveau national au travers de la toponymie cartographique. Ajoutons que la toponymie est en métropole une science active et variée, bien au-delà de l'anthroponymie origine (noms de personne attachés à des noms de lieux $)^{11}$.

Mais la prise en compte de l'importante toponymie mélanésienne et celle de son écriture ne

10. Délégation générale à la langue française et aux langues de France. Voir Bernard Cerquiglini (2003).

11. Sur l'activité toponymique en France, on visitera avec intérêt le site : http://www.ign.fr/toponymie. 
sont pas les seuls problèmes d'une toponymie qui se voudrait officielle. D'abord, la toponymie européenne évolue elle aussi et malgré les cent cinquante ans de présence française et de la prééminence de l'écrit censé stabiliser l'information, on est parfois surpris par les dérapages et les incohérences des écritures dans le temps et au travers des divers documents, des cartes terrestres et marines. On lira ainsi avec intérêt le remarquable article de Georges Pisier sur la toponymie maritime (historique) de Nouméa et ses environs (1989) ${ }^{12}$. Mais un autre problème est apparu ces dernières années : il ne suffit pas de se donner des règles (linguistiques) d'écriture, comme le demandait Jean Guiart, et comme cela a été fait avec la convention INALCO, encore fallait-il les conserver, les faire reconnaitre, pour qu'une certaine unité des écritures se dégage, dans l'intérêt général et le souci de pérenniser l'information recueillie. Or, il faut bien constater que rien n'a été fait dans ce sens et c'est toujours l'originalité et la spécificité de la transcription toponymique qui priment dans les nombreux travaux publiés ou en cours. En réalité, c'est un vieux démon qui a toujours collé à la peau des Occidentaux, qu'ils soient découvreurs $^{13}$, chercheurs ou scientifiques, sans oublier naturellement les responsables directs de la « cartographie officielle ». Faut-il aussi parler des transcriptions aberrantes comme dans la Ouinné, le col des étiquettes (des sondages miniers), traduit par le col aux biquettes, à la Madeleine, le coldes Ovatas (nom latin d'une plante), traduit par le col des Ouata. Terminons avec les fautes d'orthographe, comme celles relevées avec humour par un botaniste sur la signalisation routière et publiées dans les Nouvelles calédoniennes ${ }^{14}$ : le Trou aux Peruches, l'Hermitage, l'Immaculé Conception, le creek Abscinthe, l'école La Farendole.

En l'absence de commissions officielles, d'organismes compétents reconnus et de convention officielle d'écriture unique et reconnue pour la rédaction cartographique des langues mélanésiennes, il faut bien reconnaître qu'en NouvelleCalédonie, la toponymie n'est pas encore été considérée comme une science (officielle). Tout y est permis, aujourd'hui et plus que jamais, au grand dam des usagers de plus en plus perplexes et déroutés par les évolutions en cours et la multiplicité des écritures au travers des différentes cartes et documents et dont la presse se fait régulièrement écho :
«Faut-il parler de nouveau de chaos ou de grand bazar?»

Par ailleurs, la toponymie et la sensibilisation aux langues vernaculaires, comme la connaissance d'une convention d'écriture cartographique générale, devraient faire l'objet d'un enseignement systématique et renouvelé, auprès de toutes les personnes ayant à manipuler de l'information géographique ou ayant des responsabilités en la matière. Compte tenu de la diversité de nos langues, on peut aussi imaginer sur les cartes un petit lexique des langues de la région couverte.

Il n'est jamais trop tard pour bien faire, et la mise en place d'une commission de toponymie permanente locale, ouverte à tous, en particulier aux responsables publics concernés, paraît une priorité et une évidence pour que la « continuité du Service public» ne soit pas qu'un simple slogan de circonstance.

\section{La toponymie et l'accord de Nouméa}

Si cette action en toponymie de 1983 à 1993 a largement anticipé la prise en compte du patrimoine toponymique mélanésien, l'accord de Nouméa de 1998 est venu y ajouter la touche politique spécifiant que «les noms kanak des lieux seront recensés et rétablis ». La loi organique de 1999 a suivi pour la redistribution des rôles (compétences). Parmi les instances officielles, la première à réagir sera la province Nord qui lancera ses actions en 2002. Axée sur la collecte du patrimoine kanak, elle s'appuiera cependant sur la base de données toponymiques du ST-NC qu'elle « complètera, corrigera » mais semble-til, sans réelle coopération avec le ST-NC ni continuité avec les travaux antérieurs, dont la convention INALCO de 1990 ce qui ne fera que perpétuer l'instabilité des écritures. Une nouvelle signalisation routière, " en langue », en sera l'illustration médiatique la plus spectaculaire. Bien que répondant à l'accord de Nouméa, c'est oublier les obligations légales qui devraient normalement être attachées à cette matière, ainsi que les aspects spécifiques de l'approche cartographique. En 2003, l'ADCK ${ }^{15}$ avec le Centre culturel Tjibaou poursuivront les actions de collecte culturelle et fin 2005, ils recevront une commande de la province Sud pour une nouvelle signalisation routière, en double écriture, avec la valorisation culturelle et touristique de certains toponymes.

12. Toponymie reprise dans le catalogue de l'exposition La Ville en Dessin (et Dessein) de 1997-1998.

13. On lira à ce propos les observations d'Hélène Blais (2005: 210-215).

14. Les programmes de la Société ne mentionnent aucun concert le 5 juin 1873.

15. Agence de développement de la culture kanak. 


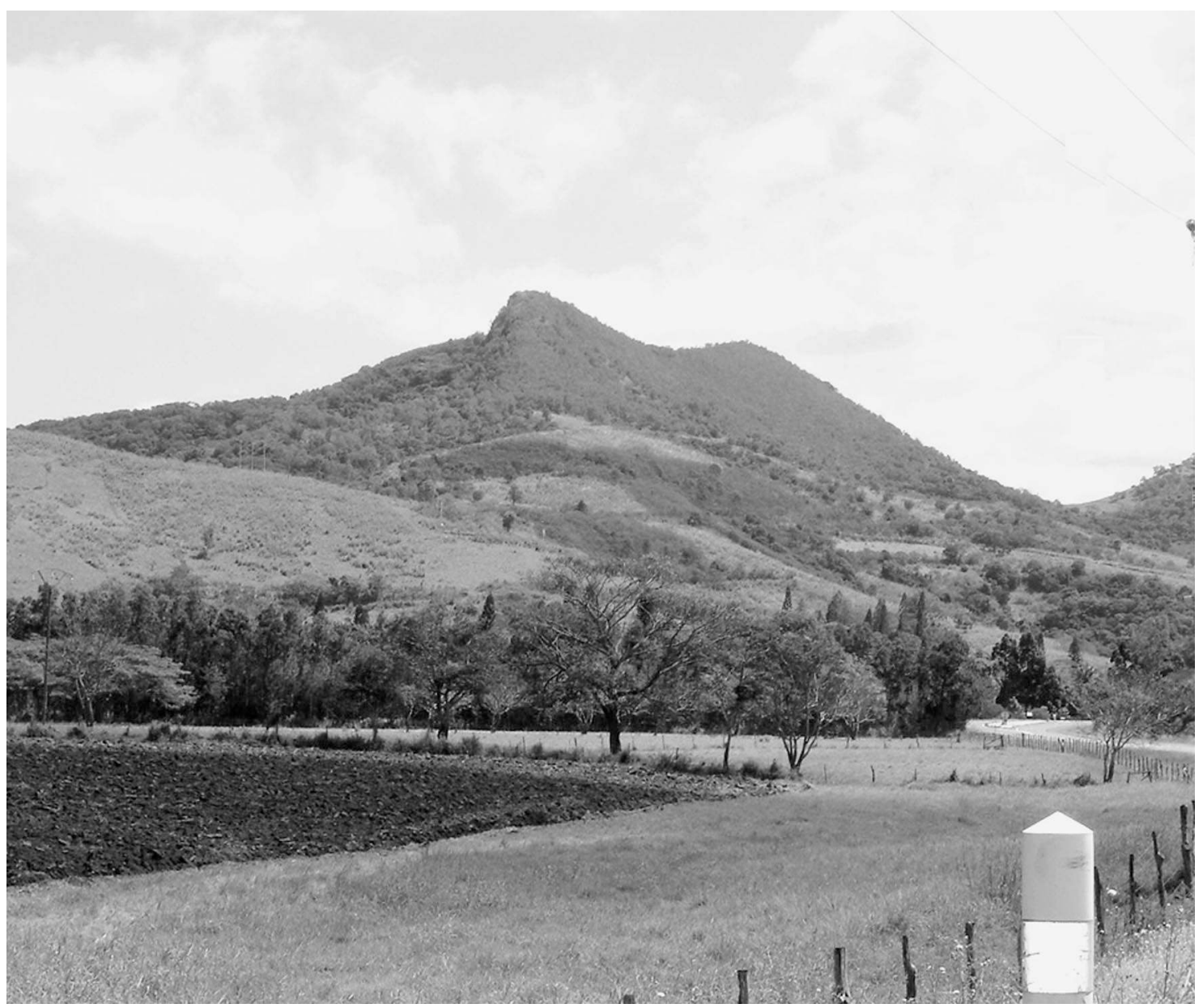

FIGURE 8. - Le Kocétorekoa, un site bien connu. La signification serait « briser le cou ». C'est là que, sur ordre du grand chef, l'on précipitait de la falaise les hommes et les femmes qui avaient commis des fautes graves envers la chefferie ou dans la population. (G. Païta, 2006)

Le programme doit s'étaler sur quatre années pleines et la mise en route débutera à la fin de l'année 2006. L'équipe ADCK a été étoffée en 2005 et fera appel aux géomètres du secteur privé.

Mais il a surtout été décidé dorénavant de collaborer avec le ST-NC. Cette nouvelle approche, plus rigoureuse et scientifique qu'en province Nord, conjuguant les aspects culturels et cartographiques, pourrait constituer un enrichissement réciproque intéressant. En respectant quelques règles (simplicité et homogénéité d'écriture sur la base d'une convention unique et officialisée), ces actions pourraient conduire à une triple reconnaissance du patrimoine toponymique et sa conservation en stabilisant les écritures :

1. au niveau de la Nouvelle-Calédonie par les commissions communales, avec la prise en compte de la toponymie générale traditionnellement reconnue par tous, en double information s'il y a lieu ;

2. au niveau de la France, par la Commission nationale française ;

3. au niveau international et des pays francophones par cette même commission.

Le classement en cours de parties de nos récifs au Patrimoine de l'humanité va certainement lui aussi, ranimer l'intérêt pour la toponymie et c'est ce que la province des Iles est en train de faire, et probablement les autres provinces. Restera donc à trouver un fédérateur pour que ce patrimoine soit cohérent sur toute la Nouvelle-Calédonie, à moins qu'on ne revienne à la culture de nos différences et au chaos originel, mais cette foisci, singulièrement amplifié.

Bien du chemin reste à faire et le rassemble- 
ment tous azimuts des compétences et bonnes volontés ne sera pas de trop sur ce sujet bien délicat, au travers de notre population multiraciale et de notre évolution politique en cours. Souhaitons que la voix de la raison et de la sagesse soit entendue.

\section{BIBLIOGRAPHIE}

BLAIs Hélène, 2005. Voyage au Grand Océan (géographie du Pacifique et colonisation, 1815-1845), Paris, éditions du Comité des travaux historiques et scientifiques (CTHS).
BouRgEY Lieutenant,1864 (25/12). Voyage d'exploration de Ounia à Boulari, Moniteur de la NouvelleCalédonie 274.

Cerquiglini Bernard, 2003. Les langues de France, Paris, PUF.

Guiart Jean, 2002. Et le masque sortit de la mer, Nouméa, Le Rocher-à-la-Voile.

PISIER Georges, 1989. La toponymie maritime (historique) de Nouméa et ses environs, Bulletin de la Société des études historiques de Nouvelle-Calédonie 79 , pp. 5-23. 